\title{
Biodiversity Indexes: Value and Evaluation Purposes
}

\author{
Jatna Supriatna ${ }^{1}$ \\ ${ }^{1}$ Institute for Sustainable Earth and Resources (I-SER) and Dept of Biology, Faculty of Math and Sciences, \\ Universitas Indonesia
}

\begin{abstract}
Biodiversity is a word recently introduced by experts in the field of biology. This word became more meaningful after Edward O. Wilson of Harvard University introduced it in a book entitled Biodiversity, an extension of biological diversity, in 1989 [11]. In subsequent developments, it became very popular and used not only by environmental biologists but also by researchers, environmentalists, funders, educators, social experts, economists, policy makers, and many others, although many do not know what that means. Biodiversity includes variations within the biological community, where living species, and ecosystems, where communities are located, as well as interaction between them (Pri. The science of biodiversity has emerged rapidly since then included monitoring and evaluation systems which is measuring the value of biodiversity components, such as the number of species present, the population of species, a habitat or the sum of all such components within a given area or site. Such monitoring and evaluation may be carried out for a variety of reasons, included identification of a given area for biodiversity richness, evenness or healthy ecosystems. The richness is the number of species per sample, the more species present in a sample, the richer the sample. Evenness is a measure of the relative abundance of the different species making up the richness of an area. Two commonly used to measure biodiversity Simpson index Ds and Shannon's index H'. Simpson's index DS is similarity index (the higher the value the lower in diversity). While Shannon index is combining evenness and richness and less weighted on dominant species. Both indexes are more reflective in nature and can predict the environment health. Therefore, it may be good to have one of those biodiversity indexes to be used for UI GreenMetric to understand the environment healthiness in the campus.
\end{abstract}

\section{Introduction}

When the UI GreenMetric chairperson asked me how to do we measure biodiversity in the campus, of course, I have so many things in my mind. As a professor who has taught Conservation Biology for the last 3 decades at UI, I have so many possible explanations to measure biodiversity, either scientifically measured that has to be carried out by biologists or other model that may be friendly for anyone in the campus who can fill in the form as long as they know what kind of biodiversity locally known. Therefore, I took the liberty to propose by describing biodiversity first then what model need to be used in measuring biodiversity in the campus.

Green Campus is varied in many ways. Local university's outdoor-education program, local bike paths connect more to the city area, fishing, beach walking, disc golf, triathlon, shooting sports team, rowing, sailing, and more traditional sports are found mostly in the open spaces of the campus. Those are open spaces created by most of the university campuses all over the world. However, some city campus, of course, will be difficult to get more open spaces for those activities. Those many open spaces hosted full with biodiversity in it. But we need to understand which biodiversity we may measure, for example whether we count common species, richness or endemic biota, or proportion of those then we call it an index.
In this short paper, I want to share what should UI GreenMetric do in adding biodiversity on its formula. There is not going to be easy to use those indexes with mathematical equation unless we develop a computational tool that may be user-friendly by even small campuses. Therefore, I recommend having a new formula instead the current index in order to get more holistic view on biodiversity such as included richness, endemicity or native, representativeness, and others.

\section{What is biodiversity and why we measure.}

The term of biodiversity is not so long only a few decades ago when the person who come up with the term of biological diversity then to be shortening to become biodiversity. Although the science of flora, fauna and small creatures have been recognized as oldest one, biology, but the genuine term was proposed by Edward O Wilson of Harvard University in his paper in 1985 entitled "Biological diversity crisis" then a book called Biodiversity in 1989 [10, 11]. Soon after than book called Conservation Biology written by Michael Soule (1996) used biodiversity in many chapters [7]. It becomes very popular worlds after UN Earth Summit in 1992 at Rio de Janairo Brazil. The summit results many world's agenda to tackle the environmental problems 
included; Agenda 21, Forest for People, Climate Change and Convention on Biological Diversity (CBD). This CBD convention has been developing many commitments to conserve biodiversity in every 4 years meetings. Those commitments are now becoming a major driving force behind efforts to reform land management and development practices worldwide and to establish a more harmonious relationship between people and nature [8].

With working definition of biodiversity as the variety of life and its processes, it means including the variety of living organism, the genetic differences among them, the communities and ecosystems in which they occur [11]. The importance of this definition is that it draws attention to the many dimensions of biodiversity. It explicitly recognizes that every biota can be characterized by its taxonomic, ecological, and genetic diversity and that the way these dimensions of diversity vary over space and time is a key feature of biodiversity. Thus, only a multidimensional assessment of biodiversity can provide insights into the relationship between changes in biodiversity and changes in ecosystem functioning and ecosystem service. Biodiversity is the foundation of ecosystem service to which human well-being is intimately linked. No feature of Earth is more complex, dynamic, and varied than the layer of living organisms that occupy its surfaces and its seas, and no feature is experiencing more dramatic change at the hands of humans than this extraordinary, singularly unique feature of Earth. This layer of living organisms - the biosphere - through the collective metabolic activities of its innumerable plants, animals, and microbes physically and chemically unites the atmosphere, geosphere, and hydrosphere into one environmental system within which millions of species, including humans, have thrived. It follows that largescale human influences over this biota have tremendous impacts on human well-being. It also follows that the nature of these impacts, good or bad, is within the power of humans to influence [1].

There are many measures on biodiversity; species richness (the number of species in a given area) represents a single but important metric that is valuable as the common currency of the diversity of life-but it must be integrated with other metrics to fully capture biodiversity. Because the multidimensionality of biodiversity poses formidable challenges to its measurement, a variety of surrogate or proxy measures are often used. These include the species richness of specific taxa, the number of distinct plant functional types (such as grasses, forbs, bushes, or trees), or the diversity of distinct gene sequences in a sample of microbial DNA taken from the soil. Species- or other taxon-based measures of biodiversity, however, rarely capture key attributes such as variability, function, quantity, and distribution - all of which provide insight into the roles of biodiversity [2].

Ecological indicators are scientific constructs that use quantitative data to measure aspects of biodiversity and ecological condition, services, or drivers of change, but no single ecological indicator captures all the dimensions of biodiversity. Ecological indicators form a critical component of monitoring, assessment, and decisionmaking and are designed to communicate information quickly and easily to policy makers [3]. In a similar manner, economic indicators such as GDP are highly influential and well understood by decision makers. Some environmental indicators, such as global mean temperature and atmospheric $\mathrm{CO}_{2}$ concentrations, are becoming widely accepted as measures of anthropogenic effects on global climate. Ecological indicators are founded on much the same principles and therefore carry with them similar pros and cons [4].

\section{Measuring biodiversity by index}

Many indices have been applied on environmental issues. Those are the Living Planet Index (LPI), Ecological Footprint (EF), City Development Index (CDI), Human Development Index (HDI), Environmental Sustainability Index (ESI), Environmental Performance Index (EPI), Environmental Vulnerability Index (EVI), Index of Sustainable Economic Welfare/Genuine Progress Index (ISEW/GPI), Well-Being Index (WI), Genuine Savings Index (GS), and Environmental Adjusted Domestic Product (EDP). And Biodiversity index. The latest is not so commonly used but it is starting to get traction after Singapore made City Biodiversity index [2].

For Biodiversity index, it would be easier if biodiversity could be measured by the quantity of birds in a forest, wildflowers in a meadow or beetles in a log. Unfortunately, the simplicity is not one of the virtues of biodiversity [4]. Ecosystem are more complex then we can imagine. A common misconception is that biodiversity is equivalent to species diversity, more species in an area, it means greater its biodiversity. Biodiversity is not a number of game; the quality is more important than quantity. It is not so much in number of species but in its identity [2]. For example, fragmenting old growth forest with clear cut, would increase species richness at local scale but not contribute to species richness at a broader scale if sensitive species were lost from the landscape [4].

Diversification can all too easily become homogenization. The greatest cause of homogenization is the introduction of non-native species of plants and animals, often called exotics. Exotics are species that have invaded new areas due to accidental or deliberate transport by human. In many cities and also in the campus, those exotics have been commonly found due to deliberately planted or released it. The exotics polluted the native flora and fauna, but their contribution was nothing to biodiversity. Regions invaded by exotics lose their distinctive characters, the results is global impoverishment [4].

In spite of many tools and data sources, biodiversity remains difficult to quantify precisely. But precise answers are seldom needed to devise an effective understanding of where biodiversity is, how it is changing over space and time, the drivers responsible for such change, the consequences of such change for ecosystem service and human well-being, and the 
response options available. Ideally, to assess the conditions and trends of biodiversity either globally or sub-globally, it is necessary to measure the abundance of all organisms over space and time, using taxonomy (such as the number of species), functional traits (for example, the ecological type such as nitrogen-fixing plants like legumes versus non-nitrogen-fixing plants), and the interactions among species that affect their dynamics and function (predation, parasitism, competition, and facilitation such as pollination, for instance, and how strongly such interactions affect ecosystems). Even more important would be to estimate turnover of biodiversity, not just point estimates in space or time. Currently, it is not possible to do this with much accuracy because the data are lacking. Even for the taxonomic component of biodiversity, where information is the best, considerable uncertainty remains about the true extent and changes in taxonomic diversity [2].

When diversity indices are used in ecology, the types of interest are usually species, but they can also be other categories, such as genera, family, functional type or haplotypes. The entities of interest are usually individual plants or animals, and the measure of abundance can be, for example, number of individuals, biomass or coverage. In demography, the entities of interest can be people, and the types of interest various demographic groups. In information science, the entities can be characters and the types the different letters of the alphabet. The most commonly used diversity indices are simple transformations of the effective number of types (also known as 'true diversity'), but each diversity index can also be interpreted in its own right as a measure corresponding to some real phenomenon (but a different one for each diversity index) [3].

Many indices only account for categorical diversity between subjects or entities. Such indices, however, do not account for the total variation (diversity) that can be held between subjects or entities which occurs only when both categorical and qualitative diversity are calculated. True diversity, or the effective number of types, refers to the number of equally abundant types needed for the average proportional abundance of the types to equal that observed in the dataset of interest (where all types may not be equally abundant). Globally threatened species that have been assessed following the IUCN Red List criteria as having a high risk of extinction, restricted-range species with small global distributions, assemblages of species concerned to a particular broad habitat type, or biome and congregations of species that gather in large numbers at species sites during some stage in their life cycle [4].

\subsection{Species Richness}

Richness R simply quantifies how many different types the dataset of interest contains. For example, species richness (usually noted $\mathrm{S}$ ) of a dataset is the number of different species in the corresponding species list. Richness is a simple measure, so it has been a popular diversity index in ecology, where abundance data are often not available for the datasets of interest. Because richness does not take the abundances of the types into account, it is not the same thing as diversity, which does take abundances into account. However, if true diversity is calculated with $\mathrm{q}=0$, the effective number of types (D) equals the actual number of types (R) [2].

\subsection{Shannon and Simpson indexes}

The Shannon index has been a popular diversity index in the ecological literature, where it is also known as Shannon's diversity index, the Shannon-Wiener index, the Shannon-Weaver index and the Shannon entropy. The measure was originally proposed by Claude Shannon to quantify the entropy (uncertainty or information content) in strings of text. ${ }^{[5]}$ The idea is that the more different letters there are, and the more equal their proportional abundances in the string of interest, the more difficult it is to correctly predict which letter will be the next one in the string. The Shannon entropy quantifies the uncertainty (entropy or degree of surprise) associated with this prediction. It is most often calculated as follows [2]:

$$
\mathrm{H}=\Sigma \text { pi ln pi or } \mathrm{H}^{\prime}=-\Sigma\left(\mathrm{n}_{\mathrm{i}} / \mathrm{N}\right) * \log \left(\mathrm{n}_{\mathrm{i}} / \mathrm{N}\right)
$$

where $\mathrm{p}_{\mathrm{i}}$ is the proportion of characters belonging to the ith type of letter in the string of interest. In ecology, $p_{i}$ is often the proportion of individuals belonging to the ith species in the dataset of interest. Then the Shannon entropy quantifies the uncertainty in predicting the species identity of an individual that is taken at random from the dataset. Although the equation is here written with natural logarithms, the base of the logarithm used when calculating the Shannon entropy can be chosen freely. Shannon himself discussed logarithm bases 2, 10 and e, and these have since become the most popular bases in applications that use the Shannon entropy.

Since mean proportional abundance of the types increases with decreasing number of types and increasing abundance of the most abundant type, $\lambda$ obtains small values in datasets of high diversity and large values in datasets of low diversity. This is counterintuitive behavior for a diversity index, so often such transformations of $\lambda$ that increase with increasing diversity have been used instead [2].

The most popular of such indices have been the inverse Simpson index (1/ $\lambda$ ) [6] and the Gini-Simpson index $(1-\lambda)$. Both of these have also been called the Simpson index in the ecological literature, so care is needed to avoid accidentally comparing the different indices as if they were the same. The formula for diversity (D) is as follows:

$$
\mathrm{D}=1-\Sigma(\mathrm{n} / \mathrm{N})^{2}
$$

where $\mathrm{n}$ is the abundance of the $\mathrm{i}$-th species in an area and $\mathrm{N}$ the ii, total number of said species living in the same area.

This simply equals true diversity of order 2 , i.e. the effective number of types that is obtained when the 
weighted arithmetic mean is used to quantify average proportional abundance of types in the dataset of interest. The original Simpson index equals the probability that two entities taken at random from the dataset of interest (with replacement) represent the same type. Its transformation $1-\lambda$ therefore equals the probability that the two entities represent different types. This measure is also known in ecology as the probability of interspecific encounter (PIE) and the Gini-Simpson index [9].

\section{Other biodiversity indexes related to city}

The biodiversity areas out of species index have been used by many cities but pioneered by Singapore Government with developing formula for the so called "The city Biodiversity index" [12]. This formula may fit with the campus model (UI GreenMetric) with specific modification in the future included not only species per se but also other criteria or indicators included:

1. Proportion of natural area in the city

2. Connectivity measure or ecological networks to counter fragmentation

3. Native biodiversity in built up area (bird species)

4. Change number of native species

5. Proportion of protected area

6. Proportion of exotics or invasive species

7. Regulation on quantity of water use

8. Climate regulation, carbon storage and cooling effects

9. Recreational and education

10. Budget allocated for biodiversity

11. Number of biodiversity projects annually

12. Policies, rules and regulations: existence of Biodiversity Strategic action Plan

13. Institutional capacity

14. Participation and partnership

15. Education and awareness

Those indicators have already developed a user manual so that proposed cities can use it in order to see whether they are can make a planned against the indicators.

The criteria for identifying key biodiversity areas need to be agreed upon internationally backbone university at UI GreenMetric and these standards must be consistently applied. However, to guarantee ownership of the indicator plans and the sustainability of efforts to implement them, the process of identifying these indicators must be led at a national or international levels.

\section{References}

1. Groom, M.J., K.K. Meffe, R. Caroll. Principles of Conservation Biology. Sinauer Associates, Icn, Sundeland, M.A (2006)

2. Hill, D., M. Fashmam, P. Shaw, Handbook of Biodiversity methods: Survey, Evaluation and Monitoring, Cambridge University Press, Cambridge (2005)

3. Noss, R.F., Indicators for monitoring biodiversity: $A$ hierarchial. Conser, Biol 4: 355-364 (1990)

4. Noss, R.F \& A.Y. Cooperrider, Saving nature's legacy: Protecting and Restoring Biodiversity,. Island Press, Washington, D.C. (1994)

5. Primack, R., Primer of Conservation Biology. Sinauer Associates, Sunderland, M.A. (2004)

6. Simpson, E. H., "Measurement of diversity", Nature. 163: 688. doi: 10.1038/163688a0 (1949)

7. Soule, M., Conservation Biology: the Science of Scarcity and Diversity, Sinauer Asscoaites, Sunderland, M.A. (1986)

8. Supriatna, J., Melestarikan alam Indonesia, Yayasan Obor, Jakarta (2009)

9. Tuomisto, H., "A consistent terminology for quantifying species diversity? Yes, it does exist", Oecologia. 4: 853-860. doi:10.1007/s00442-0101812-0 (2010)

10. Wilson, E.O., The Biological Diversity Crisis, Bioscience 35: 700-706 (1985)

11. Wilson, E.O., The current state of biological diversity. In: E.O. Wilson (ed): Biodiversity, pp3-18, National Academy Press, Washington, D.C. (1989)

12. www.cbd.int/.../city/.../subws-2014-01-singaporeindex. 